\title{
Retinochoroidal toxoplasmosis in a patient with cerebral post-transplant lymphoproliferative disease of Hodgkin's type: a diagnostic challenge
}

\author{
Ruchi Mittal ${ }^{1}$, Gabrielle Thumann², George Souteyrand ${ }^{2}$, David Kuerten ${ }^{3}$ and Sarah E. Coupland ${ }^{4}$
}

\begin{abstract}
Toxoplasmosis is a relatively rare complication in renal transplant patients and can pose diagnostic challenges, especially when it manifests as an ocular inflammation. Authors hereby report an unusual case of a 57-year-old male who developed retinochoroidal toxoplasmosis after 15 years of renal transplant, the diagnoses of which were challenging as the patient was also a known case of cerebral post-transplant lymphoproliferative disease (PTLD) of Hodgkin's type, which misled the ophthalmologists towards a clinical diagnosis of ocular PTLD. Histopathology examination of the enucleated eye revealed numerous toxoplasmosis cysts within the retina and choroid. No ocular PTLD was observed.
\end{abstract}

Keywords: Retinochoroidal toxoplasmosis; Renal transplant; Post-transplant lymphoproliferative disease; Retinal necrosis; Toxoplasmosis infection; Hodgkin's disease; Uveitis; Oculocerebral; Inflammation; Histology

\section{Correspondence}

Although reported previously, retinochoroiditis due to toxoplasmosis is relatively rare in renal transplant patients [1]. A definitive timely diagnosis in such cases can be challenging, particularly in atypical clinical presentations, as in our patient, who was also diagnosed with cerebral post-transplant lymphoproliferative disease (PTLD) of Hodgkin's type [2,3]. The presentation of bilateral extensive uveitis due to retinochoroidal toxoplasmosis 15 years after renal transplantation, but clinically suspected to be an oculocerebral PTLD, has not been reported previously to our knowledge.

\section{Case description}

A 57-year-old male presented to Aachen University Eye Clinic in June 2009 with bilateral uveitis and vitritis. He had undergone a renal transplant for chronic glomerulopathy in 1990 and had received immunosuppression with cyclosporin A, mycophenolate mofetil and fortecortin. He suffered episodic uveitis in the left (OS) and right

\footnotetext{
* Correspondence: S.E.Coupland@liverpool.ac.uk

${ }^{4}$ Liverpool Ocular Oncology Research Group, Department of Molecular and Clinical Cancer Medicine, Institute of Translational Medicine, University of Liverpool, 6th Floor Duncan Building, Daulby Street, Liverpool L69 3GA, UK Full list of author information is available at the end of the article
}

(OD) eyes in 2003 and 2006, respectively (Fig. 1). On both occasions, vitreous biopsies did not reveal definitive infectious causes (e.g. viral or protozoa) in either real time-polymerase chain reaction (RT-PCR) analyses or in bacterial and fungal cultures. Furthermore, atypical cells were not observed within the reactive lymphocytic infiltrates on microscopy. Serological examinations ruled out an infectious aetiology at this stage. On both occasions, vancomycin was administered intraocularly, whilst cefuroxime was given systemically. This led to a resolution of the ocular inflammation and ultimate development of focal retinochoroidal scars without evidence of hyperpigmentation in both eyes.

In 2007, the patient developed right-sided leg weakness and paralysis. Computerised tomographic (CT) scans revealed a left frontal lobe lesion. A stereotactic biopsy was performed, and a rare cerebral PTLD of Hodgkin's type was diagnosed, positive for CD30 and Epstein Barr virus (EBV)-encoded latent membrane protein-1 (Fig. 2a-d). Importantly, there was no histological evidence of dual pathology-i.e. there was no evidence of microorganisms. The patient was treated with external beam radiotherapy (40 Gy) in divided doses.

In March 2009, the patient developed a rhegmatogenous retinal detachment OD, arising in the area of the 

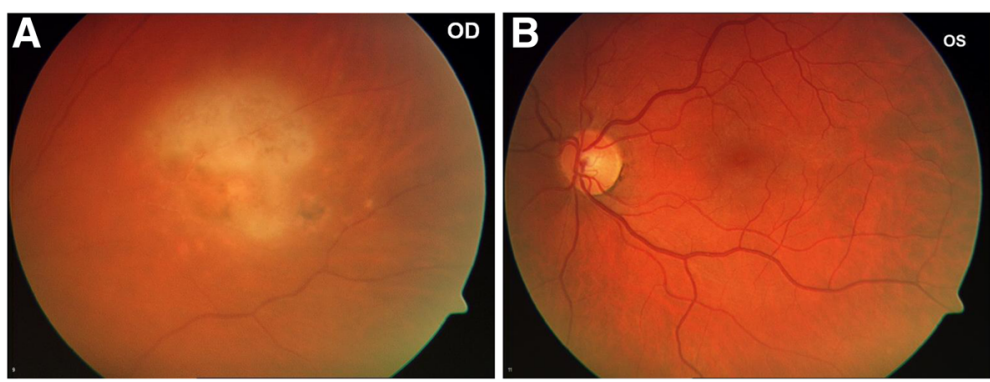

Fig. 1 Colour fundus photographs of the right $\mathbf{a}$ and left $\mathbf{b}$ eyes, taken in 2006

residual retinochoroidal scars, which did not show any signs of reactivation; a vitreous biopsy performed at that time did not reveal any further information. On examination in June 2009, the patient had light perception only OD with a raised intraocular pressure (IOP) of $23 \mathrm{mmHg}$ and a visual acuity of $0.1 \mathrm{OS}$. On fundoscopy, there were numerous extensive yellowish white retinochoroidal infiltrates OS, extending to the peripapillary region (Fig. 3a). In view of the previous history of cerebral PTLD, a clinical diagnosis of presumed oculocerebral PTLD was made, and the treatment option with intravitreal methotrexate was considered; however, confirmation of clinical diagnoses was required. Since the OD was nearly blind and painful with a raised IOP of $23 \mathrm{mmHg}$, and considering that the previous vitreous biopsy was non-informative, the right eye was enucleated.

Histological sections demonstrated an enlarged globe with focal scleral thinning. Moderately dense chronic inflammatory infiltrates were seen in the anterior chamber, iris stroma, ciliary muscles, anterior vitreous and entire choroid, with focal vasculitis (Fig. 3b). The retina was near-to-completely necrotic (Fig. 3c, asterisk marked) with the remaining viable areas being detached. Focal areas of retinal scarring, with RPE proliferation at the scar edges, were seen (Fig. 3d). Within the necrotic retina, numerous toxoplasmosis cysts were observed (Fig. 3e, arrows); these were confirmed on immunohistochemistry (Fig. 3f). There

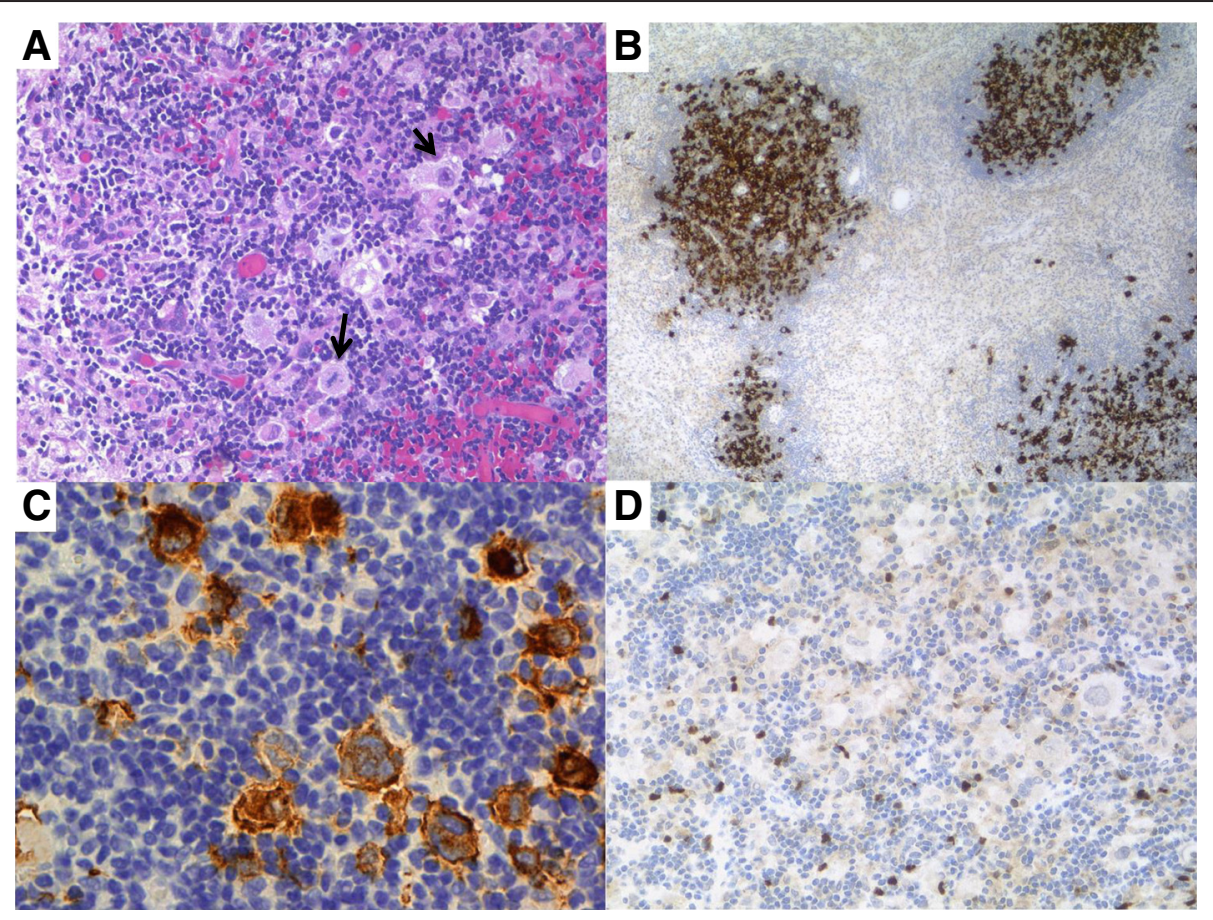

Fig. 2 a Photomicrograph of the cerebral biopsy showing numerous mononuclear Hodgkin cells and multinucleated Reed Sternberg (HRS) cells in a cellular background (arrows) (haematoxylin-eosin; original magnification $\times 200$ ). HRS cells are positive for b CD30 (PAP immunostaining; original magnification $\times 100$ ) and strongly express the $\mathbf{c}$ Epstein Barr virus-encoded latent membrane protein-1. d BOB.1 is negative in the HRS cells and is positive in bystander cells (PAP immunostaining; original magnification $\times 400$ ) (Images courtesy of Prof. I. Anagnostopoulos) 


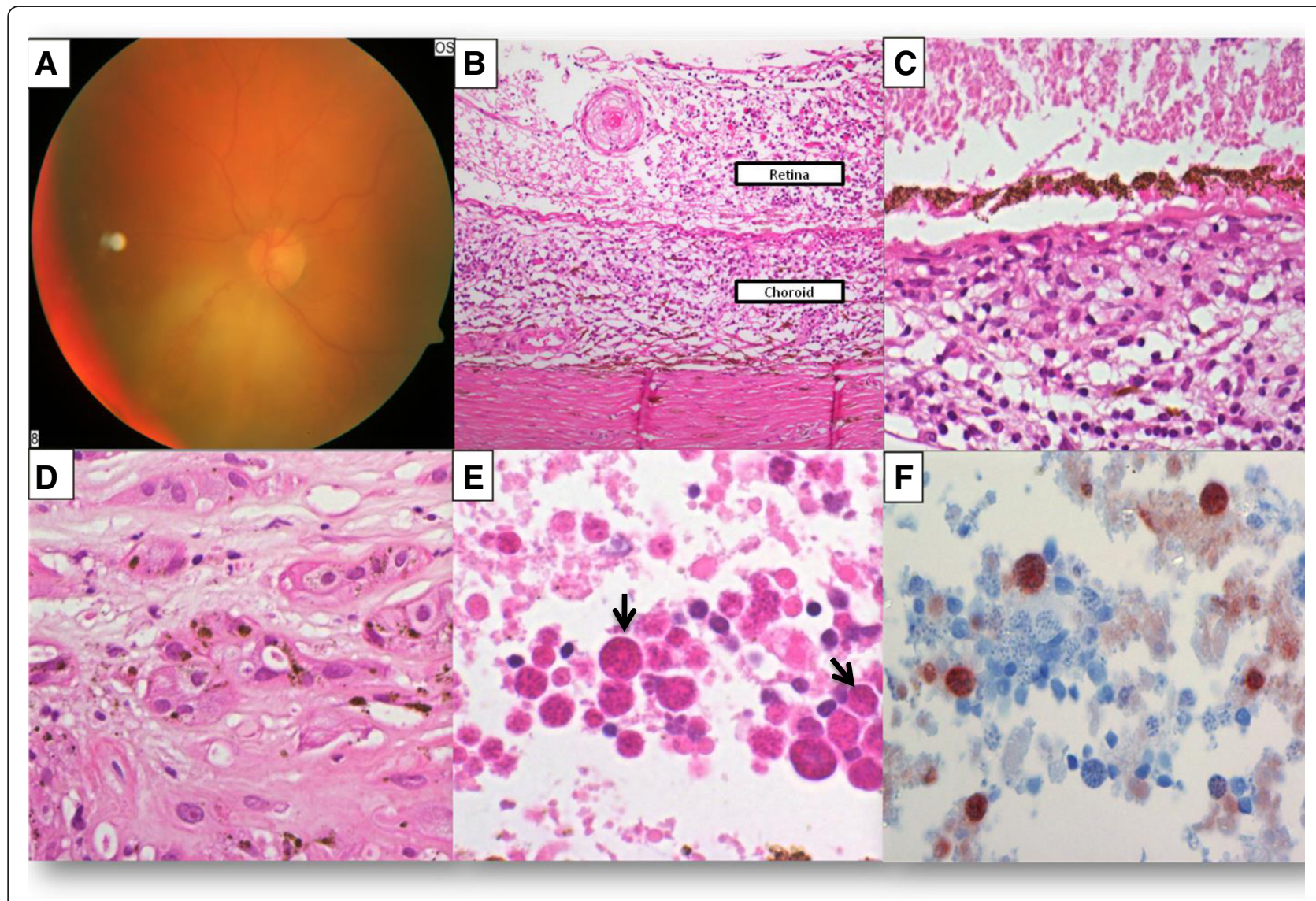

Fig. 3 a Colour fundus photograph of the left eye shows extensive yellowish white retinochoroidal infiltrates. b Photomicrograph shows chronic inflammation of the choroid and retina (haematoxylin-eosin; original magnification $\times 100$ ), with c retinal necrosis (haematoxylin-eosin; original magnification $\times 200$ ). $\mathbf{d}$ Scarring of the retina with hyperplasia of retinal pigment epithelium was noted focally (haematoxylin-eosin; original magnification $\times 400$ ). e Necrotic retina shows numerous cysts of toxoplasma (arrows) (haematoxylin-eosin; original magnification $\times 1000$ ). $\mathbf{f} C y s t s$ are positive for antibodies to Toxoplasma gondii (PAP immunostaining; $\times 1000)$

was no evidence of any Hodgkin-like CD30+/EBV+ cells despite numerous levels being performed. A diagnosis of extensive chronic uveitis with necrotic retinochoroiditis caused by Toxoplasma gondii was made. Subsequent cerebrospinal fluid and serum examinations were positive for T. gondii by PCR and enzyme-linked immunosorbent assay. This contrasted subsequent cerebral biopsies, which showed neither toxoplasmosis cysts nor residual PTLD cells. Furthermore, the blood toxoplasma IgG levels were only slightly elevated, and IgM was negative. The patient received treatment with combination of pyrimethamine, sulfadiazine and clindamycin; however, he unfortunately succumbed to the combined disease processes shortly thereafter.

\section{Discussion}

This case illustrates a number of unusual pathologies and clinical challenges. Firstly, it describes a case of cerebral PTLD of Hodgkin-type, which has been never reported in the literature. Secondly, it exemplifies the fact that an open mind must be kept in the differential diagnosis of immunosuppressed patients with a "masquerade syndrome"-like presentation. Although the main clinical suspicion in the present case was intraocular involvement of the cerebral PTLD, the differential diagnosis of intraocular toxoplasmosis was equally highly ranked, considering the medical history of the patient. Fulminant toxoplasmosis in post-transplant patients may occur as a primary infection shortly after transplantation or as reactivation of latent infection, even up to 7 years after transplantation [4]. It may have variable uveal involvement and can progress rapidly to panophthalmitis and even orbital cellulitis [5]. Hence, dual pathologies coexisting in a single patient, possibly at the same [6] or different sites, must always be considered, and early diagnosis of these entities, although challenging, is essential.

\section{Abbreviations}

EBV: Epstein Barr virus; IOP: intraocular pressure; OD: right; OS: left; PTLD: post-transplant lymphoproliferative disease. 


\section{Competing interests}

The authors declare that they have no competing interests.

\section{Authors' contributions}

GS and GT were responsible for the clinical diagnoses and management of the patient. SEC carried out the laboratory investigations, histomorphological diagnoses of the case, data acquisition, drafting and revision of the manuscript. RM participated in the data acquisition, drafting and revising of the manuscript. DK aided in the revision of the manuscript. All authors read and approved the final manuscript.

\section{Authors' information}

GT and GS are clinical ophthalmologists at the Hôpitaux Universitaires de Genève. GT is also Head of the Posterior Ophthalmological Service in Geneva.

DK is a clinical Ophthalmologist at the University Hospital in Aachen. SEC is the George Holt Chair of Pathology at the Institute of Translational Medicine, Lead Ocular Pathologist at the RLBUHT, and Lead of the LOORG research group (www.loorg.org).

RM is a Consultant pathologist at L.V. Prasad Eye Institute and is a Visiting Fellow at the University of Liverpool, UK, sponsored through the University of Liverpool-India Fellowship programme (2014-2015).

\section{Acknowledgements}

The authors would like to thank Mr Simon Biddolph, Biomedical Scientist and Advanced Practitioner in Ophthalmic Pathology at the Royal Liverpool University Hospital, for the excellent histological sections provided of the case presented.

\section{Author details}

'Dalmia Ophthalmic Pathology Services, L.V. Prasad Eye Institute, Bhubaneswar, India. ${ }^{2}$ Département des neurosciences cliniques, Service d'ophthalmologie Unité du segment posterieur, Hôpitaux Universitaires de Genève, Geneve, Switzerland. ${ }^{3}$ Department of Ophthalmology, RWTH Aachen University, Aachen, Germany. ${ }^{4}$ Liverpool Ocular Oncology Research Group, Department of Molecular and Clinical Cancer Medicine, Institute of Translational Medicine, University of Liverpool, 6th Floor Duncan Building, Daulby Street, Liverpool L69 3GA, UK.

Received: 25 January 2015 Accepted: 29 July 2015

Published online: 04 August 2015

\section{References}

1. Filloy A, Garcia-Garcia O, Fernández-Lorente L (2013) Chorioretinitis as the first sign of acquired toxoplasmosis transmitted from donor following kidney transplantation: case report and review of the literature. Ocul Immunol Inflamm 21:34-35

2. Swerdlow SH, Webber SA, Chadburn A, Ferry JA (2008) WHO classification of tumours of haematopoietic and lymphoid tissues, 4th edn. IARC Press, Lyon, France, p 349

3. Choi JH, Ahn MJ, Oh YH, Han SW, Kim HJ, Lee YY, Kim IS (2006) Epstein-Barr virus-associated Hodgkin's disease following renal transplantation. Korean J Intern Med 21:46-49

4. Wulf MW, van Crevel R, Portier R, Ter Meulen CG, Melchers WJ, van der Ven A, Galama JM (2005) Toxoplasmosis after renal transplantation: implications of a missed diagnosis. J Clin Microbiol 43:3544-3547

5. Butler NJ, Furtado JM, Winthrop KL, Smith JR (2013) Ocular toxoplasmosis II: clinical features, pathology and management. Clin Experiment Ophthalmol 41:95-108

6. Mentzer A, Perry M, Fitzgerald N, Barrington S, Siddiqui A, Kulasegaram R (2012) Is it all cerebral toxoplasmosis? Lancet 21:379, 286 